\title{
Herbal Medicine News Update
}

\section{Tea drinkers live longer}

Date: January 9, 2020

Source: European Society of Cardiology

Summary: Drinking tea at least three times a week is linked with a longer and healthier life, according to a new study.

\section{Caffeine may offset some health risks of diets} high in fat, sugar

Date: December 20, 2019

Source: University of Illinois at Urbana-Champaign, News Bureau

Summary: In a study of rats, scientists found that caffeine limited weight gain and cholesterol production, despite a diet that was high in fat and sugar.

Information collected by:

Md. Akbar Hossain

Department of Pharmacy

ASA University of Science and Technology Bangladesh
3. Experts review evidence yoga is good for the brain

Date: December 12, 2019

Source: University of Illinois at Urbana-Champaign, News Bureau

Summary: Scientists have known for decades that aerobic exercise strengthens the brain and contributes to the growth of new neurons, but few studies have examined how yoga affects the brain. A review of the science finds evidence that yoga enhances many of the same brain structures and functions that benefit from aerobic exercise.

References: www.sciencedaily.com 